\title{
Simplified Strategies for COVID-19 Surge Management in Critical Care in Resource-Limited Areas - An Aide-Mémoire
}

\author{
Mehraj Ud Din ${ }^{1}$, Prashant S Hegde ${ }^{2}$
}

Purpose of this document

Coronavirus disease 2019 (COVID-19) is an infectious disease caused by the severe acute respiratory syndrome (SARS) coronavirus 2 . We are facing an unprecedented situation globally because of the COVID-19 pandemic where extraordinary measures are needed continually on war footing. The goal being to save maximum lives while protecting health care staff.

A competent emergency response system must incorporate necessary resources, relevant knowledge, attitudes and skills. Ring-fencing by clustering patients, experts and pooling of resources will go a long way in containing the pandemic locally.

While detailed disaster response policies and guidelines are available from different societies and national bodies, this concise paper presents an aide-mémoire in a simplistic manner, since clarity of thought is key to effective preparation and sustained response. [1-5]

Based on a review of various guidelines and literature search, key considerations are presented in a concise format to facilitate chalking out a suitable response strategy locally. [6-10]

The following five key areas should be considered in the hospital/institution while planning for increasing Critical Care capacity to cope with anticipated COVID-19 surge.

\section{Structure \\ 2. Staffing \\ 3. Space \\ 4. Supplies and equipment \\ 5. Sustainability and Staff Well being}

Structure (Management \& Chain of

Command)

Anticipate:

- There is going to be a sudden peak and surge in COVID-19 patients with many requiring Intensive Care Unit (ICU) admission requiring organ support particularly mechanical ventilation which will overwhelm existing health care facilities.

\section{Blueprint:}

- Establish a central/regional ICU COVID19 coordination center with dedicated and expert man power, which will guide and monitor all the ICU admissions and resource allocation in the entire region while maintaining continual contact with networking hospitals.

- Lay down and circulate policies and guidelines on different aspects of patient care to standardize management while minimizing risk to health care staff.

- Lay down clear directives for emergency upscaling of ICU beds, a simple plan for the same can be found in the document below.

- Guidelines available from WHO/Intensive Care Societies should be used for local policy making

\section{Connect / Network:}

- The central ICU coordination committee should daily connect (using Tele conferencing for instance), with the networking hospitals for update of the current situations and resource availability and allocation.

- The central ICU coordinator should keep a regular contact with governmental agencies and relevant authorities for daily updates and feedback.

- Encourage local, national and international networking via Internet to remain updated

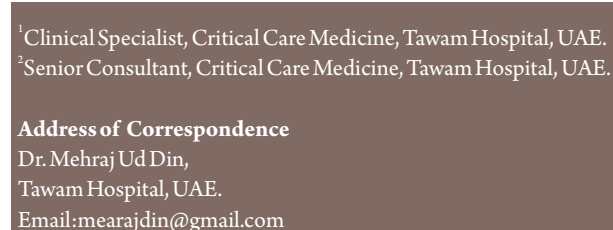

Dr. Mehraj Ud Din

MBBS, MD, DNB, EDIC, EDAIC

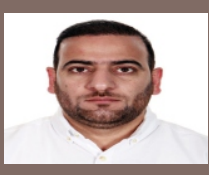

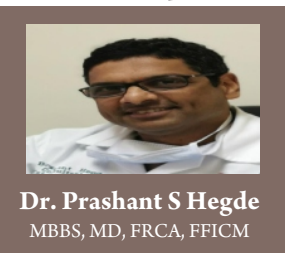

and disseminate information during the crisis.

Draft:

- Prepare a care plan protocol for critically ill COVID-19 patients using latest guidelines from WHO/Intensive Care Society (after local modification) and distribute hard copies to all networking hospitals for uniformity of care.

- Standardize the criteria for ICU referral and admission of the critically ill COVID-19 patients across the region and networking hospitals.

- Prepare standard checklists for all ICU procedures like intubations, bronchoscopy, tracheostomy, invasive vascular access. nasogastric tube insertions, proning etc. These are freely available on the web from various intensive care societies and improve quality of care.

- Prepare and formulate a clear pathway regarding aerosol generating procedures and also discuss CPR policy in COVID-19 patients which should be structured as per locallaw and beliefs.

- Print standardized information leaflets for high alert ICU medications like vasopressors and sedatives to increase patient safety. This will reduce human errors especially when non-ICU staff are recruited during the surge.

\section{Educate:}

- Educate with simulation and mock drills for familiarity in preparation for managing patients safely in a challenging environment.

- Expert team must do regular sessions for training and demonstration of using of Level 3 and Level 4 personnel protection equipment like N95, Powered Air Purifying Respirators (PAPR) etc.

- Regular webinars, telemedicine and video demonstrations of the skills and procedures is always helpful. Video demonstrations of donning and doffing PPEs are possibly more effective than printed material.

(C) 2020 by Journal of Anaesthesia and Critical Care Case Reports|Available on www.jaccr.com | DOI: 10.13107/jaccr.2020.v06i01.145

This is an Open Access article distributed under the terms of the Creative Commons Attribution Non-Commercial License (http://creativecommons.org/licenses/by-nc/3.0)

which permits unrestricted non-commercial use, distribution, and reproduction in any medium, provided the original work is properly cited. 


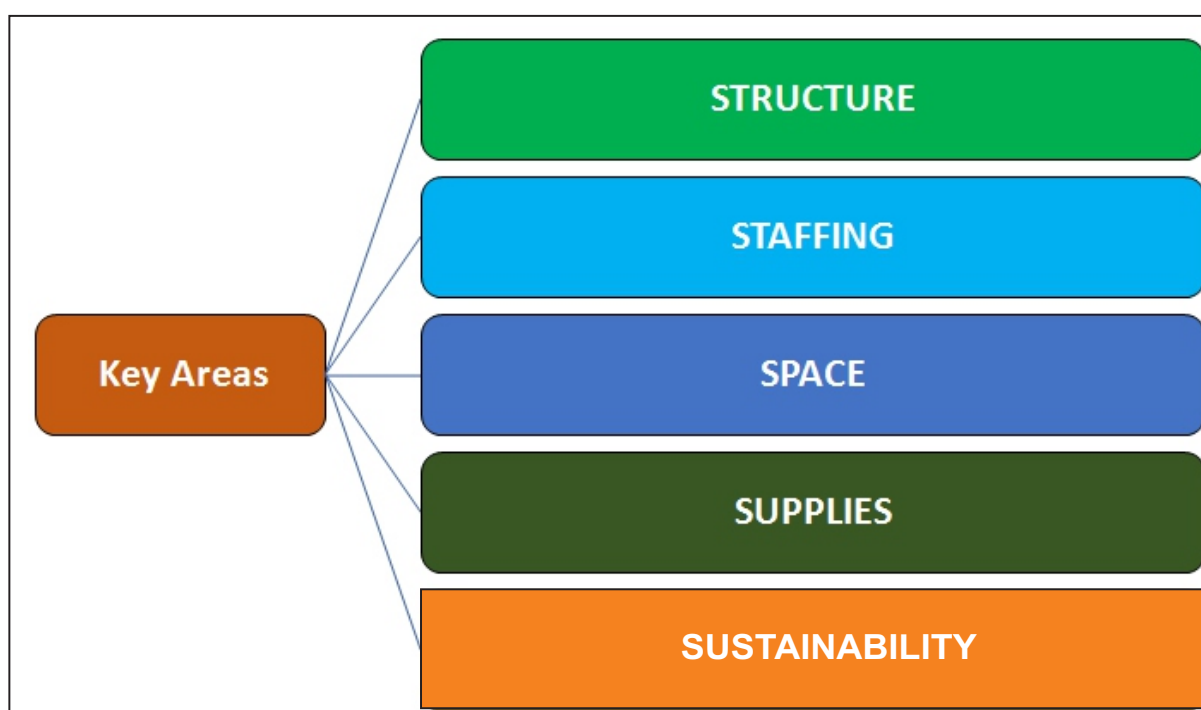

Figure 1:Five key areas to be considered during the planning for increasing the Critical Care capacity to cope with anticipated COVID-19 surge.

Formulate and Implement:

- Formulated guidelines and protocols for ICU care of COVID-19 patients with strict implementation during clinical practice will standardize patient care and improve patient safety.

- Prepare hard copies of various checklists, procedural policies and guidelines in a simplified way and distribute to networking hospitals.

- An infection control team of adequately trained nurses and doctors should supervise the training and implementation of infection control measures with regular monitoring and feedback.

- N95 mask-fit testing for all healthcare staff caring for COVID-19 patients must be done including proper donning and doffing simulations. This is critical. Losing staff to isolation or disease will lower staff morale and strain the manpower resource.

Update:

- Update the local situation daily and assess the needs and responses on day to day to basis by the nodal officers with regional ICU coordination center.

- As the knowledge and research about COVID-19 is getting released everyday there is constant need to update and review the policies and guidelines to reflect latest scientific practice.

\section{Staffing \& Manpower}

\section{ICU Physicians}

- Assign a nodal officer in each ICU, which may be head of the department or consultant ICU who will coordinate with networking hospitals and central ICU command.

- Enlist and maintain a register of all the available intensivists and physicians with relevant experience for example medicine physicians etc, in the entire region. - Inform the doctors about the situation and anticipated manpower crisis in ICU care and prepare them mentally with relevant updates and training support so that they are ready by the time need arises.

- Involve internal medicine, emergency medicine, pediatrics and other specialties in ICU rotation to give them some ICU experience and knowledge beforehand so that they are ready in dire circumstances.

- Internal medicine physicians can look after high dependency unit patients who may not require highly complex critical care.

- Remember the need for surgery, obstetrics and pediatric support during preparation phase.

\section{Nursing Staff}

- Enlist and maintain a register of all the available nurses with relevant critical care experience in the entire region.

- Collect information and contact details and start informing them about the potential ICU nursing crisis and need for ICU nurse staffing on urgent basis.

- Depute non-ICU nurses from other areas of the hospital to ICU for upscaling training and orientation by assigning them to ongoing ICU Rota under the supervision of experienced ICU staff.

- Involve nursing coordinators from each ICU to ensure proper care and reporting to anesthesiologists, pulmonologists and acute nursing supervisor and central ICU command.

- Consider pooling of critical care nurses at designated centers.

Paramedical and Allied Healthcare Workers

- Allied health care staff like ICU technicians, respiratory therapists, anesthetic technicians, pharmacists, dieticians and physiotherapists must be similarly considered during manpower planning and training.

- Involve anesthesia technicians and other health care staff who are currently not in ICU and train them for ICU care immediately by including them in the current ICU rota.

- Plan for surge in laboratory tests, blood bank requirements, (minimise radiology).

- Plan for ancillary staff like housekeeping and porters who will be needed in large numbers.

- Depute them to ICU rotation of current ICU Rota for supervision and training and quick updates and familiarity with equipment and personal safety.

- Educate and train the allied healthcare staff regarding infection control and personnel protective equipment.

Space

- Assess and formally quantify the available ICU resources for patient care and normal occupancy rate of ICU beds and create a registry for the same.

- Gather and document information about all the ICU beds available in the region both private and government setups and inform the relevant administration regarding the same.

- Identify and label the hospitals in the entire region, which will take up these patients at regional and sub-regional level and assign a nodal officer in charge for each one of them.

- Allocate and prepare a triage area in anticipation of impending crisis and identify an area with oxygen facilities in hospitals where mechanical ventilators can be used for large number of patients (for e.g.: Identify areas like emergency department, Operating rooms etc.).

- Involve the hospital engineering staff to identify and create new negative pressure isolation rooms as much as feasible on emergencybasis.

- During the ongoing pandemic ICU will be required for non-COVID-19 patients also (trauma,sepsis and other critical conditions needing organ support). Isolate areas in the 
hospital for Non-COVID-19 patients requiring ICU care.

- Consider shutting down operating rooms and converting them into makeshift ICU rooms (using High Efficiency Particulate Air filters (HEPA filters) in non-negative pressure rooms) as most of equipment can deal with mechanical ventilation and ICU monitoring.

- Test plan with mock drills and dry runs to identifyloopholes.

- Inform and anticipate that makeshift ICU can be established at district hospital levels by pooling ICU/anesthesia staff of the district at these centers after providing appropriate equipment and support.

- Appropriate facilities and spaces for natural breaks (on call rooms/rest rooms/staff room) will ensure smooth functioning and continuity of patient care.

\section{Supplies and equipment}

- Identify and prepare adequate ICU equipment including ventilators, monitors, accessories, beds BiPAP machines, high flow oxygen cannulae, crash carts with defibrillators, laryngoscopes (video laryngoscopes) and portable ultrasound machines.

- Order surplus supplies in advance as much as possible and consider pooling equipment at assigned places.

- HEPA filters should be considered as alternatives for lack of negative pressure rooms.

- BiPAP/CPAP machines can be very effective and helpful for oxygenating non COVID-19 patients requiring respiratory support in ward areas to spare ICU beds.

- Oxygen supply will be constrained during the pandemic surge. Ensure sufficient oxygen supply with full reserves and consider liquid oxygen storage.

- Encourage 'out of box common sense thinking' to optimise resource usage, for example; servicing old equipment lying in storage and making it functional as much as possible.

- Review policy and appropriately train ward staff in order to start accepting stepdown of more nonCOVID-19 high dependency patients requiring noninvasive ventilation who are otherwise hemodynamically stable, to facilitate ICU bed flow.

- Plan for dedicated transport equipment monitors, ventilators, trolleys, stretchers and transport ventilators. Minimise patient movement for investigations and discourage use of stethoscope if possible.

- Involve pharmacists and allied staff like dieticians to start stocking essential ICU supplies and medicines. Identify a team and assign responsibilities including coordination.

- Ensure adequate supply of disposable items including PPEs, gloves, gowns, masks, hand sanitizers etc.

- Limit hospital services to life threatening and limb threatening cases only in order to streamline resources during the crisis.

\section{Sustainability and staff wellbeing}

- The COVID-19 is pandemic is potentially like a marathon hence it is important to maintain a slow and steady pace where possible. There will be a need for sustained efforts and supply of resources.

- Due consideration should be given for ensuring a continuous supply of personal protective equipment.

- Staff well-being to prevent burn out is high priority to ensure the continuity of quality patient care.

- Rotation and shifts of the staff should be thoroughly planned to minimize exhaustion of the staff as much as possible.

- Consider spacing the critical care staff schedule in such a way that there is always some staff ready in case of loosing staff to exposure or illness.

- Managers must maintain culture of encouraging staff to take adequate rest and eat healthy with adequate hydration and exercise to relieve stress. Support staff with mentoring or using buddy system.

- Involve clinical psychologists for debriefing, counselling and interacting with staff to minimize the stress and anxiety while working in a high risk environments.

- Minimize red-tapism and unnecessary paperwork in a time of increased workload (keep it simple!)

- Diligently document what was done (since this will guide future planning and improvements).

\section{References}

1. https://www.who.int/emergencies/diseases/novel-coronavirus-2019/technicalguidance/critical-preparedness-readiness-and-response-actions-for-covid-19

2.https://www.sccm.org/disaster/covid-19-icu-preparedness-checklist

3. https://www.who.int/docs/default-source/documents/publications/hospital-emergencyresponse-checklist.pdf

4.Wax RS. Preparing the Intensive Care Unit for Disaster. Crit Care Clin. 2019;35(4):551-562. doi:10.1016/j.ccc.2019.06.008

5. Seda G, Parrish JS. Augmenting Critical Care Capacity in a Disaster. Crit Care Clin. 2019 Oct;35(4):563-573. doi:10.1016/j.ccc.2019.06.007.Epub2019Jul 26

6. Liao, $x$., wang, b. \& kang, $y$. Novel coronavirus infection during the 2019-2020 epidemic: preparing intensive care units-the experience in sichuan province, china. Intensive care med46,357-360(2020).https://doi.org/10.1007/s00134-020-05954-2

7. Xie, J., Tong, Z., Guan, X. Et Al. Critical Care Crisis And Some Recommendations During The Covid-19 Epidemic In China. Intensive Care Med (2020). https://Doi.Org/10.1007/S00134-020-05979-7

8. Adams jg, walls rm. Supporting the health care workforce during the covid-19 global epidemic. Jama.Published online march 12, 2020.Doi:10.1001/jama.2020.3972

9.Grasselli G, Pesenti A, Cecconi M. Critical Care Utilization for the COVID-19 Outbreak in Lombardy, Italy: Early Experience and Forecast During an Emergency Response. JAMA. Publishedonline March 13,2020. doi:10.1001/jama.2020.4031

10. Qiu, H., Tong, Z., Ma, P. et al. Intensive care during the coronavirus epidemic. Intensive Care Med 46, 576-578 (2020).https://doi.org/10.1007/s00134-020-05966-y
Conflict of Interest: Nil

Source of Support: None

\section{How to Cite this Article}

Din MU, Hegde PS. | Simplified Strategies for COVID-19 Surge Management in Critical Care in Resource-Limited Areas - An Aide-Mémoire | Journal of Anaesthesia and Critical Care Case Reports |Jan-April 2020; 6(1): 6-8. 